\title{
MEJORA CONTINUA DE LA CALIDAD EN UNA UNIDAD MÉDICA DE VALORACIÓN DE INCAPACIDAD TEMPORAL
}

\author{
ÁLVAREZ THEURER, ESTHER *; \\ VAQUERO ABELLÁN, MANUEL **
}

(*) Unidad Médica Valoración Incapacidades (UMVI).

Delegación Provincial de Salud Córdoba.

(**) Universidad de Córdoba.

\begin{abstract}
RESUMEN
Objetivo: Nuestro objetivo es el diseño de un plan de mejora continua de la calidad en una Unidad Médica de Valoración de Incapacidades (UMVI).
\end{abstract}

Métodos: La metodología utilizada sigue las directrices marcadas por la Consejería de Salud, en cuanto a procesos e indicadores, que se recogen en el Plan Anual de Inspección. En nuestra Unidad estamos implantando el modelo EFQM de Excelencia.

Resultados: Se expone nuestra propuesta de Plan de Calidad para la UMVI.

Conclusiones: Destacamos la falta de formación de los profesionales sanitarios responsables de la prescripción de la prestación de IT. Existe unanimidad respecto a la necesidad de unificar criterios de gestión de la Incapacidad Temporal, por lo que sería interesante consensuar este documento con el resto de Unidades Médicas de Valoración de Incapacidades que realizan su trabajo en la Comunidad Autónoma Andaluza, pudiéndose extender a otras Autonomías.

\section{PALABRAS CLAVES}

Mejora continua, plan de calidad, incapacidad temporal..

\section{ABSTRACT}

Objective: Our objective is design a continuous improvement quality plan in the Medical Unit for Disabilities Valuation (MUDV).

Methods: The used methodology follows the guidelines marked by the Health Council, as for processes and indicators that are picked up in the Annual Plan of Inspection. In our Unit we are implanting the EFQM pattern of Excellency.

Results: Our proposal of Quality Plan is exposed for the MUDV.

Conclusions: We highlight the lack of the sanitary professionals' responsible for the benefit prescription of the of Temporary Disability (DT) formation. Unanimity exists regarding the necessity of unifying approaches to administrate the DT. It would be interesting staying according to this document with other Medical Units for Disabilities Valuation that carries out its work in the Andalusia Autonomous Community, being able to extend to other Autonomies.

\section{KEY WORDS}

Continuous improvement, quality plan, temporary disability. 


\section{INTRODUCCIÓN}

Este documento pretende afrontar un Plan de Calidad en la Unidad Médica de Valoración de Incapacidades de Córdoba (UMVI) que garantice su función básica: el control sanitario de la prestación por IT, a la vez que mejore la atención al usuario dando prioridad a la satisfacción de sus necesidades, basándonos en las líneas estratégicas y programas específicos de los dos Planes Andaluces de Calidad y en los distintos Planes Anuales de Inspección de Servicios Sanitarios.

Con ello, pretendemos conseguir la mejora de la calidad en la prestación sanitaria de la Incapacidad Temporal, a través de un grado de implicación y dedicación importante de todos los profesionales de la Unidad Médica de Valoración de Incapacidades de Córdoba. Todo ello redundará en una mejor asistencia, y también en la relación y satisfacción de los usuarios (1).

La mejora continua de nuestros Servicios Sanitarios, como reza el lema de nuestro II Plan Andaluz De Calidad (2): "Caminando hacia la Excelencia", es una visión nueva en la cultura de la Organización en la que todos debemos participar de forma activa.

Referencias a la calidad y su gestión ya se realizan en la Ley 14/1986, de 25 de abril, General de Sanidad, y en la Ley 16/2003 de 28 de mayo, de Cohesión y Calidad del Sistema Nacional de Salud (3).

En el Primer Plan de Calidad del Sistema Sanitario Público de Andalucía, para el periodo el 20002004 (4), se define el ciudadano como centro del sistema sanitario, y su satisfacción como elemento irrenunciable de la calidad. El Segundo Plan de Calidad, para el periodo el 2005-2008, consolida las políticas de calidad anteriores, impulsando la innovación y modernización del sistema sanitario, generando un modelo de gestión de la información y creando cauces de participación del ciudadano.

Uno de los objetivos de la Inspección de Servicios Sanitarios de la Junta de Andalucía (5), en concordancia con la estrategia contenida en el Plan Director de Organización para la Calidad de los Servicios, es la mejora continua de la calidad. Persigue alcanzar el mayor grado de calidad en los servicios que presta; así como su máxima eficiencia, de modo que la tutela, control y evaluación que se realicen en la prestación sanitaria de la Incapacidad Temporal (IT) contribuya a garantizar el cumplimiento de los derechos y deberes de los ciudadanos con el Sistema de Salud (6).
En el Plan de Inspección de Prestaciones y Servicios del año 2004 se hace referencia en el apartado III.8 a la necesidad de confeccionar un Plan de Calidad en el que se contemplará un Plan de Mejora a la atención del Usuario en el ámbito de la Inspección y Control de la Incapacidad Temporal, que será desarrollado por la UMVIs.

En el Plan de Inspección de Prestaciones y Servicios del año 2005 en el apartado III.8 se expone que a lo largo del ejercicio 2005 la Subdirección de Inspección de Servicios Sanitarios dará continuidad al Plan de Calidad en el que se contempla un Plan de Mejora a la atención del Usuario en el ámbito de la Inspección y Control de la Incapacidad Temporal, que será desarrollado por la UMVIs, y que incluirá:

- Acceso vía pagina Web a información general y normativa básica en materia de Incapacidad Temporal.

- Acceso a información personal del usuario a través página Web mediante la utilización de Firma Digital.

- Establecimiento de un servicio de Información en las UMVIs.

- Mejora del sistema de cita previa en las UMVIs.

- Acciones de mejora en la formación de Incapacidad Temporal del personal de Atención Primaria y UMVI.

La Junta de Andalucía publica en el BOJA del 11 de febrero de 2005 el ACUERDO de 8 de febrero de 2005, del Consejo de Gobierno, por el que se aprueba el Plan General de Inspección para la Mejora de la Calidad de los Servicios - 2005.

Por Orden del 23 febrero de la Consejería de Salud de la Junta de Andalucía (BOJA 17 de marzo de 2006) se ha aprobado, en este año 2006 el Plan Anual de Inspección de Servicios Sanitarios (7). Se estructura en los 3 programas generales de actuación en que se encuentra organizada la Inspección, y los correspondientes programas específicos que los desarrollan. Se ha introducido un elemento más en la estructura del plan, como son las líneas estratégicas que se han definido en el II Plan de Calidad del Sistema Sanitario Público de Andalucía, en la medida que son aplicables a las funciones que desarrolla la Inspección de Servicios Sanitarios.

La Inspección de Servicios Sanitarios está integrada en la Secretaría General Técnica de la Consejería de Salud; se estructura en la Inspección Central 
y en las Inspecciones Provinciales; y, éstas constituidas por la Dirección de la Inspección Provincial, la Coordinación UMVI, los Inspectores Médicos, Farmacéutico y Subinspectores adscritos a la misma. La Inspección Provincial depende orgánicamente de la Delegación de Salud de Córdoba y funcionalmente de la Subdirección de Inspección de Servicios Sanitarios de la Junta de Andalucía.

La UMVI en Córdoba se encuentra ubicada en la Delegación Provincial de Salud (Av República Argentina, 34). Nuestra Unidad ocupa unos $250 \mathrm{~m}^{2}$ en el ala derecha de la planta baja del edificio. Entre nuestro Personal, hay 1 Coordinadora, 3 Inspectores Médicos, 1 Médico Evaluador, 3 Subinspectores y 14 Administrativos. Personal claramente insuficiente para la labor que realizamos en toda la provincia de Córdoba.

Las principales funciones de la UMVI son (5): Actuación sobre las prestaciones del sistema de Seguridad Social, gestionados por los Servicios de Salud, en materia de contingencias comunes y profesionales (Accidentes de Trabajo y Enfermedades Profesionales); Elaboración de informes técnicosanitarios relativos a entidades colaboradoras de la Seguridad Social; la Incapacidad temporal y permanente, de conformidad con la normativa de aplicación. En definitiva, nuestra misión es colaborar en la tutela y el control de la prestación sanitaria de la IT, para conseguir el uso racional de la misma.

\section{OBJETIVO DEL ESTUDIO}

Nuestro objetivo es el diseño de un plan de mejora continua de la calidad en la Unidad Médica de Valoración de Incapacidades de Córdoba.

\section{MATERIAL Y MÉTODOS}

Desde una perspectiva práctica se trata de elaborar una guía útil para el desarrollo profesional. La metodología utilizada sigue las directrices marcadas por la Consejería de Salud, en cuanto a procesos e indicadores, que se recogen en el Plan Anual de Inspección.

En cada UMVI se ha creado un Grupo de Mejora multidisciplinar que detecta y define los problemas mediante la aplicación de herramientas de calidad y remite los resultados de los análisis y las propuestas de actuación a la Comisión de Calidad, para establecer un plan de acción (8). El Grupo de Mejora UMVI Córdoba se ha constituido desde el año 2004, convocándose su última reunión en el año 2006.
La Comisión de Calidad tiene, entre otras, las siguientes funciones: promover acciones para mejorar la calidad; elaborar el Plan de Calidad y los planes de mejora específicos; priorizar problemas; establecer Sistemas de Información y operativización del Plan

Esta Comisión está constituida por el responsable del Plan de Calidad y los Coordinadores Provinciales de las UMVIs de las ocho provincias andaluzas. El citado responsable del Plan es el Coordinador del Programa de IT. La actividad inicial ha consistido en desarrollar un análisis de procedimientos funcionales en las UMVIs, que se entregó a la Comisión de Calidad como base para el desarrollo de su actividad.

En nuestra Unidad estamos implantando el modelo EFQM de Excelencia (9) ya que es un modelo flexible y que puede utilizarse en todo tipo de organizaciones, independientemente del sector al que pertenezcan y de su tamaño. Se adapta más a nuestra organización de la Unidad Médica de Valoración de Incapacidades el modelo EFQM. Es muy interesante el proceso continuo de aprendizaje, de innovación y mejora; utilizando las ideas de todos los grupos de interés. Tratamos de alcanzar una mejora verdadera y sostenida diferenciando nuestros puntos fuertes y áreas de mejora (10).

El EFQM facilita la comparación con otras organizaciones de naturaleza similar o distinta; lo que nos puede llevar a una mejora en nuestra Unidad. Es un modelo estructurado de coherencia y sentido común; y por lo tanto de gran aplicación práctica en nuestras organizaciones (11). EFQM (European Foundation for Quality Model) es una organización que se ha dedicado a hacer tangibles los principios de la calidad total, para que sean aplicables a las organizaciones. Para ello ha desarrollado un modelo de gestión de la calidad Total o Excelencia. Es un modelo de evaluación compuesto por criterios y subcriterios para obtener los puntos fuertes y débiles de la organización; y definir planes de acción consecuentes. Básicamente se emplea para la evaluación de las organizaciones, bien por personal interno o externo, llegando a conocer cuál es su estado respecto al ideal de Excelencia empresarial, así como las oportunidades de mejora (12).

Los conceptos fundamentales de la Excelencia son: Orientación hacia los resultados, Orientación al cliente, Liderazgo y Coherencia, Gestión por procesos y hechos, desarrollo e implicación de las personas, Proceso Continuo de Aprendizaje, Innovación y Mejora, Desarrollo de Alianzas y Responsabilidad 
Social de la Organización. El modelo basado en 9 criterios que se agrupan en dos categorías: Agentes Facilitadores y Resultados. Los criterios del grupo de Agentes Facilitadores analizan cómo realiza la organización las actividades clave: liderazgo, personas, política y estrategia, alianzas y recursos y procesos. Los criterios del grupo Resultados se ocupan de los resultados que se están alcanzando: resultados en las personas, resultados en los clientes, resultados en la sociedad y resultados clave. Es importante subrayar la naturaleza dinámica del Modelo, donde la innovación y el aprendizaje potencian la labor de los agentes facilitadores, dando lugar a una mejora de los resultados. Los criterios del grupo de Agentes Facilitadores utilizan al evaluar, los elementos Enfoque, Despliegue, Evaluación y Revisión; mientras que los criterios del grupo Resultados, emplean en su evaluación el elemento Resultados.

\section{RESULTADOS}

El Grupo ha ido poniendo en marcha, siguiendo la metodología propuesta, medidas específicas para resolver determinados problemas priorizados, implantando actuaciones de mejora con un responsable designado y una fecha límite para cada actividad. Así, al dar respuesta rápida y eficaz a las necesidades, se ha fomentado la implicación y participación del personal de nuestra Unidad (13).

\section{PLAN DE CALIDAD}

\section{Introducción}

Las Unidades Médicas de Valoración de Incapacidades encuadradas dentro de la Inspección Provincial, tienen como función básica el control sanitario de la prestación por incapacidad temporal, siendo su principal objetivo detectar y evitar aquellas situaciones en las que se pueda estar dando un presunto fraude en su percepción. Este cometido debe afrontarse con unas garantías respecto a eficiencia en sus resultados consiguiendo un servicio que garantice los derechos de los usuarios, y que se preste con la máxima calidad en cuanto a la atención de los mismos (14).

\section{Política de calidad}

Misión: La misión de la Inspección de Servicios Sanitarios de la Junta de Andalucía, es la tutela y control de los centros, servicios, establecimientos y prestaciones sanitarias, en tanto que agente de la autoridad sanitaria, con el objetivo de garantizar que el funcionamiento de los mismos y las prestaciones que facilitan, cumplen los requisitos establecidos por la normativa. Tiene especial significado, el desarrollo de las funciones de inspección sobre aquellos aspectos recogidos en la Ley 2/1998, de 15 de Junio, de Salud de Andalucía, como Derechos y Deberes de los ciudadanos.

Visión: La Inspección de Servicios Sanitarios de la Junta de Andalucía, en concordancia con la estrategia contenida en el Plan Director de Organización para la Calidad de los Servicios, persigue alcanzar el mayor grado de calidad en los servicios que presta, así como la máxima eficiencia de los mismos, de modo que la tutela, control y evaluación que en los ámbitos de los centros, establecimientos, servicios y prestaciones sanitarias se efectúe, contribuya a garantizar el cumplimiento de los derechos y deberes de los ciudadanos con el Sistema de Salud (15).

A tal efecto y a través de las actuaciones previstas en los respectivos Planes Anuales de Inspección, tutelará:

- El cumplimiento de los requisitos y condiciones necesarias, así como las prestaciones y atención sanitaria que ofrecen los centros, establecimientos y servicios sanitarios de titularidad pública y privada.

- El cumplimiento de los requisitos que en materia de prestación farmacéutica dispone la normativa.

- Las prestaciones que en materia de Seguridad Social, competen a los Servicios de Salud, conforme a la normativa de aplicación.

- La adecuación de la atención sanitaria en los centros y servicios sanitarios, a las normas asistenciales y a los criterios de calidad definidos por la autoridad competente.

\section{Líneas estratégicas:}

Estas se resumen, según el Programa General de Inspección de Incapacidad Temporal en tres líneas estratégicas, cuyos programas específicos incluyen: la destinada a asumir necesidades y expectativas de la ciudadanía, con programas específicos que valoran a pacientes en situación de Incapacidad Temporal (IT) mediante protocolos de actuación específicos, y aquellos que incluyen un Plan de Mejora de Atención al Usuario; la destinada a impulsar la innovación y modernización del sistema, con programas específicos de sistemas de información (Sigilum XXI) y de transmisión electrónica de P. 9 de baja y alta laboral al INSS; y, la destinada a la línea de colaboración con otras Instituciones, cuyos 
programas específicos incluyen demanda de informes, actividades de colaboración (INSS, Sistema Sanitario Público Andaluz-SSPA- y con la Inspec- ción General de Servicios) y actuaciones extraordinarias (Figuras 1 a 4 ).

Figura 1. Programa General de Inspección: Líneas estratégicas

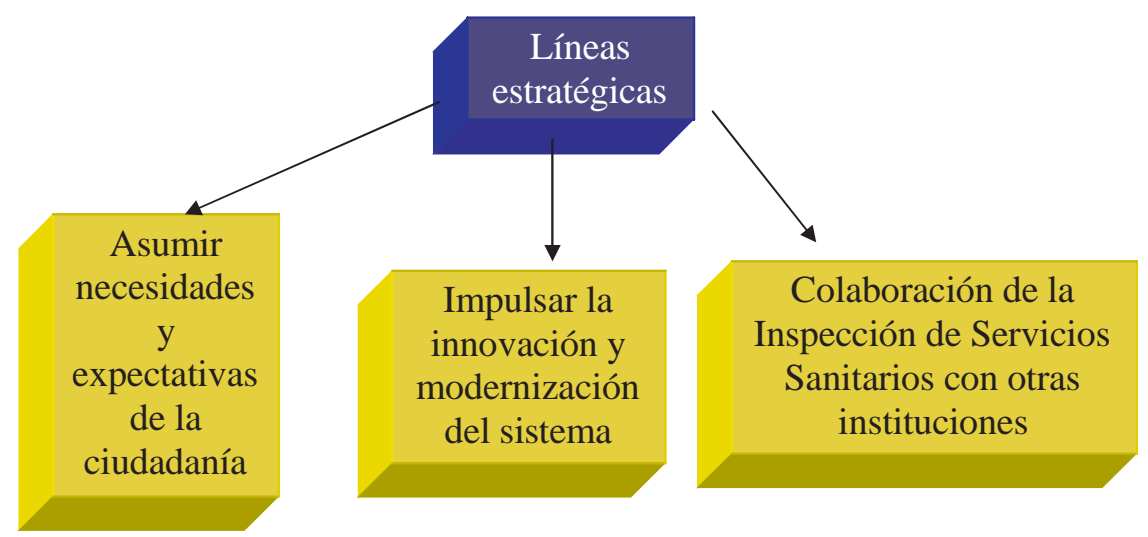

Figura 2. Programas específicos Línea estratégica: Asumir necesidades y expectativas ciudadanía.

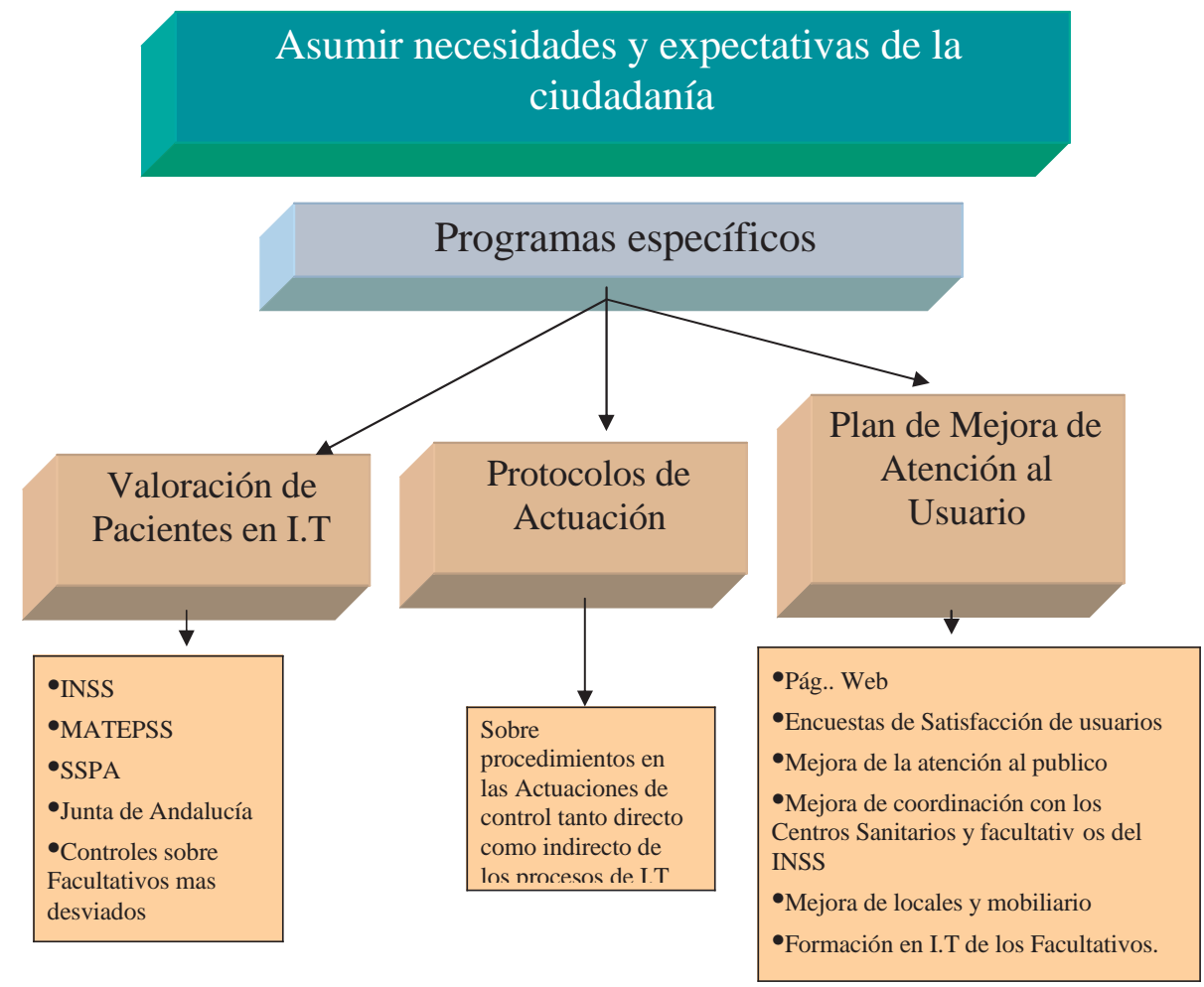


Figura 3. Programas específicos Línea estratégica: Impulsar la innovación y modernización del sistema.

\section{Impulsar la innovación y modernización del sistema}

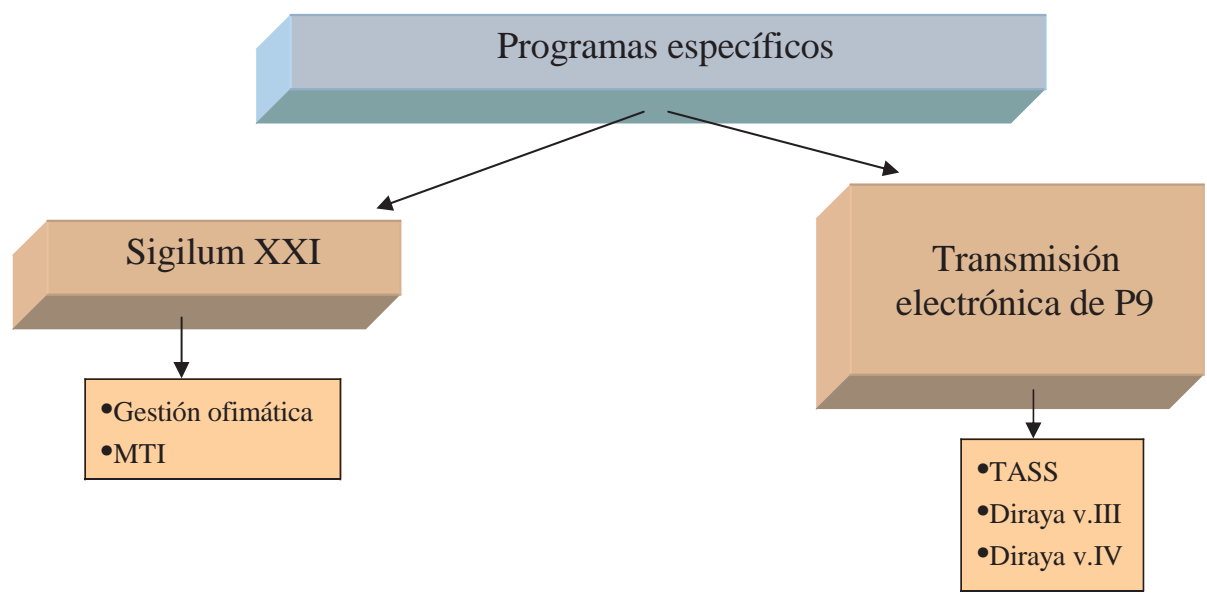

Figura 4. Programas específicos Línea estratégica: Colaboración de la Inspección de Servicios Sanitarios con otras instituciones.

\section{Colaboración de la Inspección de Servicios Sanitarios} con otras instituciones

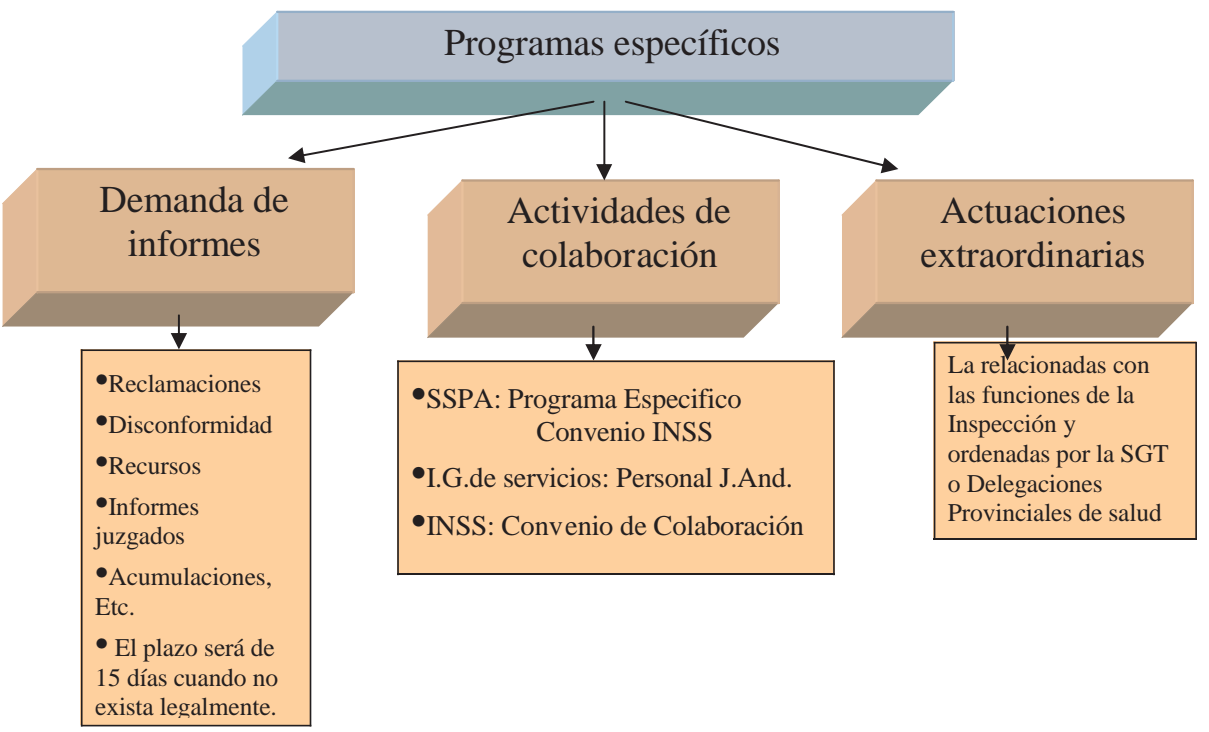




\section{Objetivo General:}

Mejora en los procedimientos de control sanitario de la prestación por Incapacidad Temporal en las UMVIS.

Atender y gestionar las relaciones entre el Sistema Sanitario Público de Andalucía y el ciudadano, que trasciende la simple consideración de este como paciente, poniendo especial énfasis en la gestión de la demanda en materia de Incapacidad Temporal, con el fin de conseguir una utilización efectiva de los distintos servicios, sobre la base de la información y la orientación al paciente-ciudadano (16).

\section{Objetivos Específicos:}

Los referidos en el plan Anual de Inspección para 2006 y adaptados por consenso en nuestra provincia.

Objetivos de Actividad y Resultados:

- Informatización: transmisión de ficheros de acuerdo al Protocolo del INSS, con un retraso máximo de 20 días

- Formación de Facultativos: Al menos del 30\% de Córdoba

- Propuesta de Alta: Plazo de contestación máximo de 15 días, desde su emisión por los facultativos del INSS o de las Mutuas.

- Disminución del gasto: conseguir el costo IT asegurado/mes establecido en convenio del INSS.

Estos objetivos figuran en el convenio de Colaboración con el Ministerio de Trabajo y las Comunidades Autónomas.

- Total asegurados revisados por Inspectores: Para su cálculo se ha multiplicado 2200 trabajadores por inspector de UMVI, lo que supone una media diaria de 10 pacientes tomando 220 días laborables al año.

- Total controles Subinspectores: se ha estimado 1000 controles de Subinspector por año.

- Propuestas de Incapacidad Permanente: 5-15\%.

- Número de asegurados revisados de la Junta de Andalucía y Mutuas: al tratarse de una actividad a demanda se ha tomado como referencia la actividad desarrollada el año 2005.

- Controles directos SSPA: Se ha tomado como referencia la prevalencia media de bajas en este personal en Andalucía, y se ha calculado el $50 \%$ de revisiones sobre todos los trabajadores en baja y se multiplica por 11 meses del año.

- $\mathrm{N}^{\circ}$ de reuniones con el SSPA: 3 anuales.

- $\mathrm{N}^{\circ}$ de visitas a centros de Atención Primaria en el control del personal del SSPA.

- Prevalencia del SSPA: La establecida para este periodo en el Programa específico de Control de I.T del Personal del SSPA.

- Incidencia del SSPA: La establecida para este periodo en el Programa específico de Control de I.T del Personal del SSPA.

- Duración media de procesos de IT del SSPA: La establecida para este periodo en el Programa específico de Control de I.T del Personal del SSPA.

- $\mathrm{N}^{\circ}$ Médicos Prescriptores evaluados: se ha tomado como referencia la posibilidad de ver al menos una vez a cada médico de la Provincia.

- Incrementar las altas sobre asegurados revisados: $35 \%$.

\section{Clientes}

Clientes internos:

- Profesionales del Sistema Sanitario Público Andaluz, tanto de Atención Primaria como de Asistencia Especializada.

- Directivos del Sistema Sanitario Público Andaluz.

- Subdirección de Inspección de Servicios Sanitarios.

Clientes externos:

- Trabajadores activos pertenecientes a cualquier Régimen de Seguridad Social.

- Funcionarios pertenecientes a Mutualidades de funcionarios como MUFACE, MUGEJU, etc.

- Las distintas Entidades Gestoras de la Seguridad Social y Colaboradoras (INSS, MATEPSS, Empresas).

- Empresas en general.

\section{Cartera de servicios}

Sistema de información:

- Codificación de partes: Asignación de un código al diagnóstico que consta en los partes de IT según codificación CIE-9 MC. 
- Elaboración de estadísticas: Recogida de los datos y agregación de los mismos para el conocimiento de la actividad desarrollada en un periodo de tiempo.

- Utilización de bases de datos y programas informáticos: Sigilum XXI y Gerhonte.

- Acceso via web: consulta por los usuarios de datos relacionados con la IT, a través de una página web.

\section{Evaluación - Control:}

- Revisión fichero de IT: Examinar datos existentes en los ficheros sobre cada proceso de IT, para determinar la actuación a seguir en cada caso.

- Revisión listados IT: revisión de listados de trabajadores en IT según criterios preestablecidos.

- Valoración del paciente: Anamnesis y/o exploración con el asegurado en IT para valoración y/o seguimiento.

- Valoración de información clínica: Solicitud y análisis de información clínica para valorar la situación del paciente en IT.

- Informe clínico laboral: Emisión de informe clínico laboral para proponer una incapacidad permanente, alta por agotamiento de plazo, alta por inspección, alta por incomparecencia...

- Contestación a empresas: Respuesta a la solicitud de información no clínica de una empresa sobre la situación de IT de uno de sus trabajadores.

- Resolución de acúmulo de periodos: Resolver si los periodos de IT son acumulables o no a efectos de agotamiento de plazo.

- Autorización de desplazamientos y traslados: Autorización de desplazamientos y/o traslados a otras localidades de trabajadores en situación de IT.

- Reclamaciones y recursos: Elaboración de un informe en los casos de reclamaciones y recursos relacionados con situaciones de IT.

- Gestión de posibles irregularidades en la percepción de la prestación de IT con puesta en conocimiento de la Autoridad u Organismos competentes.

- Visita domiciliaria: Comprobación del estado y/o situación de un paciente en IT mediante visita en su domicilio.
- Control telefónico: Comprobación del estado y/o situación de un paciente en IT mediante llamada telefónica.

- Actuaciones extraordinarias: respuesta al $100 \%$ de las actuaciones extraordinarias demandadas, dentro del plazo reglamentariamente establecido.

\section{Relación INSS - Mutuas:}

- Resolución de propuestas de alta por mejoría de Mutua: Elaboración de la resolución de las propuestas de alta por mejoría, remitidas según la legislación vigente.

- Resolución de propuestas de incapacidad permanente de Mutuas e INSS: Resolver la solicitud de una mutua o del INSS proponiendo la incapacidad permanente a un paciente.

- Resolución de intenciones de alta del INSS: Emisión de conformidad o disconformidad ante las intenciones de alta remitidas por INSS según la legislación vigente.

- Informes médicos de control de IT: Elaboración de informes trimestrales según la legislación vigente y a solicitud de los servicios médicos del INSS o Mutuas sobre situación clínica de los pacientes en IT que tienen la contingencia cubierta con ellas.

- Determinación de contingencia: Emisión de la información clínico-administrativa necesaria para que el INSS pueda determinar sobre la naturaleza común o profesional de un proceso de IT.

\section{Coordinación:}

- Asistencia al Equipo de Valoración de Incapacidades (EVI): Participación como vocal en la reunión del EVI y preparación de la misma.

- Asistencia a reuniones de coordinación INSS - Mutuas: Participación como vocal en la Subcomisión Provincial de IT.

- Asistencia a reuniones de coordinación con Mutuas: Reuniones periódicas con mutuas para tratar temas de gestión de IT.

- Asistencia a reuniones de coordinación con SSPA (Servicio Público de Salud de Andalucía): Participación en reuniones de seguimiento del programa específico de control de IT en centros del Sistema Sanitario Público de Andalucía. 
- Reuniones con equipo de Atención Primaria: Reuniones periódicas con los facultativos de Atención Primaria para control y seguimiento de los pacientes en IT.

- Asistencia a reuniones con la Inspección Provincial de Servicios: Reuniones periódicas por parte de las UMVIS con la Inspección Provincial de Servicios para el seguimiento y coordinación del Acuerdo de colaboración.

- Asistencia a reuniones con Equipo Central de Inspección: Reuniones periódicas con los coordinadores provinciales de IT para homogeneizar procedimientos en toda la comunidad autónoma y seguimiento de las actividades realizadas.

- Reuniones de la Comisión de Calidad: Reuniones periódicas del grupo de mejora multidisciplinar para establecer actuaciones a seguir en la mejora continua.

- Reuniones de Equipo: Reuniones periódicas con el equipo multidisciplinar de la UMVI con el fin de delimitar acciones encaminadas a la consecución de objetivos previstos en el Plan Anual de Inspección.

\section{Formación:}

- Información: Asesoramiento e información sobre normativa de aplicación en materia de prestación de Incapacidad Temporal, sobre procedimientos relacionados con la Incapacidad y al respecto de la situación y contenido de expedientes administrativos en relación a la IT del asegurado.

- Formación: Jornadas y reuniones formativas dirigidas a los facultativos de Atención Primaria con relación a la gestión y trámite de la Incapacidad Temporal.

\section{Procesos}

La Unidad identifica los procesos (17), como aquellos que permiten el desarrollo de las funciones y competencias que tiene asignadas y que dan respuesta a los distintos grupos de interés en el contexto de la política y estrategia de intervención definida por la Consejería de Salud. (18).

Los procesos clave en nuestra organización son:

1. Valoración del Paciente en situación de Incapacidad Temporal (IT):

Subproceso: Valoración por control directo del Paciente.
Subproceso: Valoración por control indirecto del Paciente.

2. Valoración del Paciente en situación de alta laboral.

Los procedimientos están basados en diversos protocolos de actuación:

Procedimiento de Propuesta de Alta por curación o mejoría de Mutuas.

Procedimiento de Propuesta de Alta por Incapacidad de Mutuas.

Procedimiento de Propuesta de Alta por Incapacidad del INSS.

Procedimiento de Intención de Alta por curación o mejoría del INSS.

Procedimiento de Alta por inspección: por curación o mejoría, por agotamiento de plazo máximo, por propuesta de Incapacidad, por Incomparecencia, por fallecimiento.

Procedimiento de Determinación de Contingencia.

Procedimiento de Acumulación de procesos.

Procedimiento de autorización de desplazamiento y traslado.

Procedimiento de Reclamación contra el Alta laboral.

Procedimiento de actuación en la valoración del paciente mediante visita domiciliaria.

Procedimiento de valoración a través de Informe clínico laboral para control de IT: elaboración de Informes trimestrales, según legislación vigente, en control de IT; gestión de posibles irregularidades en la percepción de la prestación de IT; elaboración de Informes clínico-laborales de control de IT, a solicitud de los Servicios Médicos de INSS, Mutuas y Empresas.

Protocolo de actuaciones de Subinspectores.

Protocolo de actuación en demanda de baja médica a Servicio de Salud tras alta por AT en Mutua.

Protocolo de actuación en IT tras baja por AT en Instituciones Penitenciarias. 
Procedimiento de mejora de los tipos y criterios de cita previa en las UMVIs.

Procedimiento para revisión de listados de asegurados en situación de IT.

\section{Indicadores y Monitorización}

\section{Indicadores Generales (19):}

Satisfacción de clientes externos e internos: a base de encuestas de satisfacción y entrevistas con ambos clientes, se obtiene dicha información.

Estudio de quejas y reclamaciones recibidas, que serán contestadas en el plazo establecido y serán objeto de revisión como procesos "centinelas" del funcionamiento de la Unidad (referentes al "funcionamiento" de la unidad, no las disconformidades con las resoluciones administrativas). Al término de cada semestre se hará un estudio sobre la evolución de las reclamaciones por unidades, motivos, tipo de profesional afectado, tiempo y tipo de respuesta.

Consentimiento informado en aquellos casos en los que se requiera para la realización de pruebas.

Cumplimentación protocolizada de historias clínico-laborales.

Los indicadores Específicos incluidos en el Plan Anual de Inspección se recogen en la tabla I.

Otros indicadores se elaboran mensualmente

Tabla I. Indicadores Específicos incluidos en el Plan Anual de Inspección.

\begin{tabular}{|c|c|c|}
\hline Indicador & Fórmula & Fuente \\
\hline $\mathrm{N}^{\mathrm{o}}$ asegurados revisados & $\mathrm{N}^{\mathrm{o}}$ total & MTI(Sigilum XXI*) \\
\hline $\mathrm{N}^{\mathrm{o}}$ asegurados revisados SSPA & $\mathrm{N}^{\mathrm{o}}$ total & MTI(Sigilum XXI*) \\
\hline $\mathrm{N}^{\circ}$ asegurados revisados Junta de Andalucía & $\mathrm{N}^{\mathrm{o}}$ total & MTI(Sigilum XXI*) \\
\hline $\mathrm{N}^{\circ}$ Facultativos entrevistados & $\mathrm{N}^{\circ}$ total & MTI(Sigilum XXI*) \\
\hline Porcentaje de altas & $\mathrm{N}^{\circ}$ de altas / $\mathrm{n}^{\circ}$ de asegurados revisados x 100 & MTI(Sigilum XXI*) \\
\hline Días tramite Propuestas de alta & $\begin{array}{l}\mathrm{N}^{\circ} \text { Prop. Pendientes a fin de mes x 30,41 / } \\
\text { promedio mensual de recibidas }\end{array}$ & MTI(Sigilum XXI *) \\
\hline Costo I.T asegurado mes & $\begin{array}{l}\text { Gasto mes / } \\
\mathrm{n}^{\circ} \text { afiliados }\end{array}$ & INSS \\
\hline Prevalencia SSPA & $\begin{array}{l}N^{\circ} \text { bajas a fin de periodo / total trabajadores } \\
\text { x } 100\end{array}$ & Gerhonte* \\
\hline
\end{tabular}


para proporcionar estadísticas del módulo MTI (Módulo de Tratamiento de la Información) del programa informático de gestión de IT, Sigilum XXI.

Con respecto a la monitorización, la Inspección Provincial dispone de sistemas de información con capacidad para garantizar la veracidad de los datos que configuran la evaluación y seguimiento del pre- sente Acuerdo. Por ello, se compromete al envío periódico de los datos para la elaboración de los Sistemas de Información y Memoria de la Inspección de Servicios Sanitarios, en los tiempos adecuados y calidad necesaria.

A modo de ejemplo, se expone la construcción de un indicador en la tabla II (20).

\section{Tabla II. Construcción de Indicador}

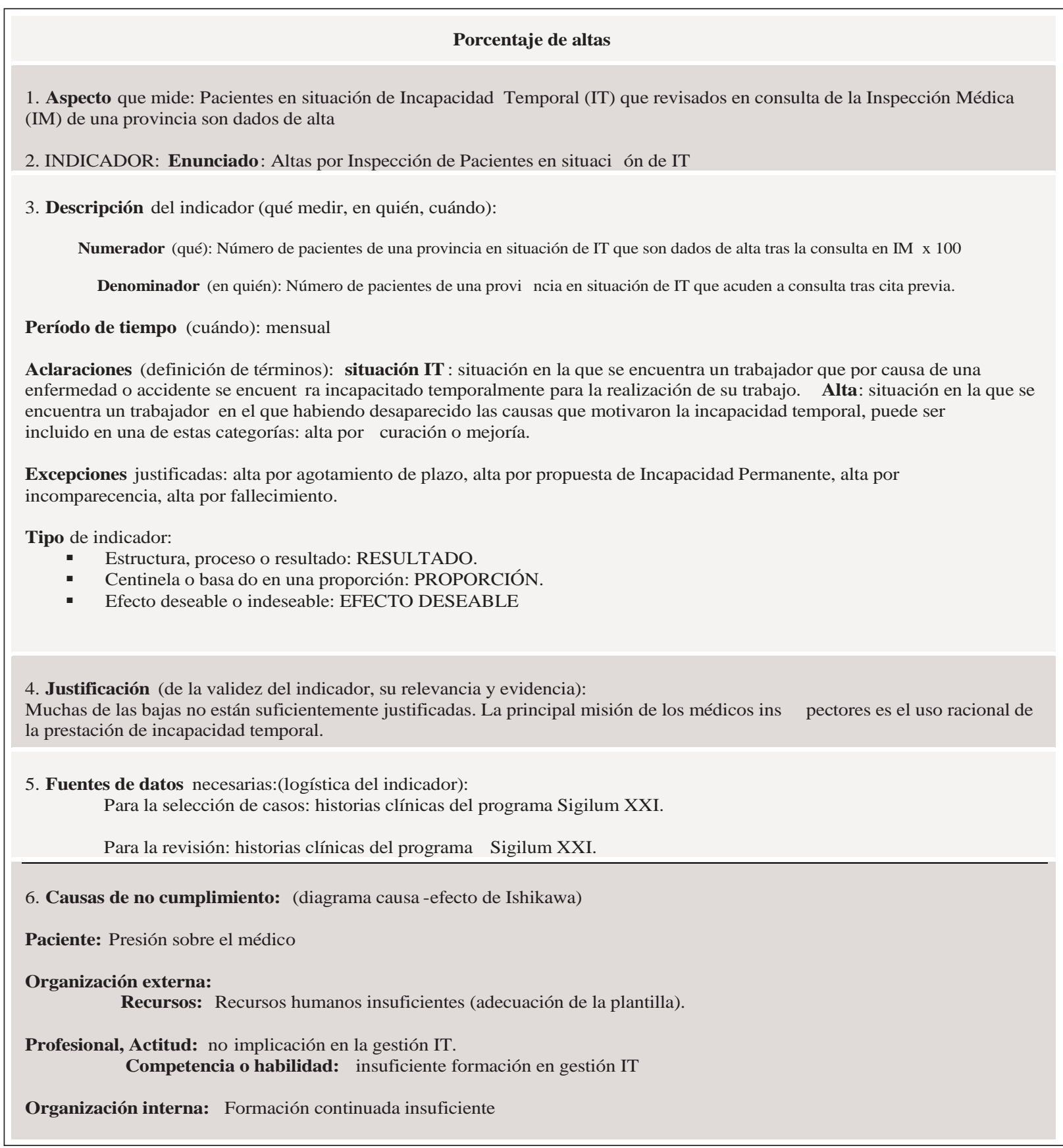




\section{Evaluación}

A cargo de la Comisión de Calidad está el establecimiento de un sistema de Evaluación Continua y seguimiento del Plan de Calidad, mediante la aplicación de indicadores (21).

Se revisan de manera sistemática y continuada los resultados obtenidos, no sólo para mantenerlos en el tiempo, sino también para ayudar a mejorar nuestro rendimiento (22).

\section{Comunicación}

\section{Comunicación externa:}

Citaciones a los pacientes-asegurados que serán vistos en consulta, a través de cartas , en ocasiones certificadas y con acuse de recibo.

Correos y comunicación telefónica con compañeros Médicos (Atención Primaria, Medicina del Trabajo y otros Inspectores del Servicio Público de Salud o del INSS) para aclarar dudas en la gestión de IT, revisiones, solicitud de informes clínicos y resultado de pruebas complementarias.

Visitas regladas a los Centros de Atención Primaria, comunicándolo previamente por teléfono y posteriormente vía fax a los Directores de los mismos. Elaboración de documentos informativos, haciendo entrega de ellos en las visitas y a través de correo postal.

Reuniones periódicas, convocadas por correo electrónico y correo ordinario, de las UMVIs con INSS, Mutuas, SSPA y la Inspección Provincial de Servicios de la Junta de Andalucía (Comisión Provincial de IT), para el seguimiento y coordinación de acuerdos de colaboración.

Comunicación con empresas que solicitan informes por escrito, en relación a la situación administrativa del trabajador asegurado.

\section{Comunicación interna:}

A través de notas interiores, cartas personales, correo electrónico y reuniones con profesionales de distintas áreas de organización de la UMVI.

\section{Formación}

\section{- Impartida a:}

Médicos de Atención Primaria, con cursos y jornadas sobre gestión de IT, además de las visitas rea- lizadas a los centros. Los médicos que asisten, se constituyen en eslabones de una cadena de formación de formadores con los compañeros de su centro de salud, potenciándose su formación en calidad (23). Desde nuestra Unidad se ha creado una plataforma virtual que pretende vía web trasladar la formación práctica a los colectivos implicados en la gestión y control de la Incapacidad Temporal: "eFormIT".

Médicos residentes en Medicina del Trabajo y Medicina de Familia, como parte de sus ciclos formativos.

- Recibida, sobre temas como:

- Comunicación y atención al usuario de Servicios de Salud

- Valoración del menoscabo en patología raquídea

- Plan de calidad en la Administración Pública Andaluza. Modelo europeo de gestión de calidad (EFQM)

- Herramientas informáticas para la Inspección Sanitaria

- Responsabilidad patrimonial: Valoración del Daño Corporal en el contexto de la Atención Sanitaria en el Sistema Sanitario Público

En cualquier caso se busca el consenso a la hora de determinar las necesidades de formación para implicar a los profesionales, conocidas sus inquietudes científicas.

\section{Investigación}

Las Unidades de la Inspección Provincial propondrán y/o participarán en los proyectos de investigación, relacionados con las funciones de la Inspección de Servicios Sanitarios, que se propicien desde la Inspección Central. De igual modo participarán en las actividades de formación organizadas para mejorar la adecuación de los profesionales a los puestos de trabajo que desempeñan. Por otro lado potenciarán publicaciones en jornadas, congresos, revistas, etc.

Nuestra UMVI está desarrollando un proyecto de investigación de la Consejería de Salud de la Junta de Andalucía. Hemos publicado, en revistas indexadas, trabajos científicos. En los dos últimos años se han presentado comunicaciones en Congresos Nacionales, recibiendo en dos de ellos el premio a la Mejor Comunicación. 


\section{DISCUSION}

Este trabajo pretende ser un documento de ayuda para los profesionales que realizamos nuestra labor en una Unidad Médica de Valoración de Incapacidades y servir de base para el desarrollo de un proceso de mejora continua (16).

La principal dificultad se halla en conseguir la implicación de todos los profesionales; dada la escasez de recursos humanos que presenta la UMVI, fundamentalmente en número de Inspectores Médicos, unido al ingente quehacer diario. Recientemente, hemos conseguido -tras la labor realizada - que se contrate un Inspector médico más en nuestro equipo.

Otra limitación importante que aparece en la priorización de problemas, y que no depende de nosotros, es la incompleta implantación del Programa informático gestión IT (Sigilum XXI). Esto se subsanará en un futuro inmediato y facilitará nuestra labor en la gestión de IT.

Es necesaria la coordinación entre todos los agentes que participan en una eficiente gestión de la prestación de IT. Ello contribuirá a mejorar la calidad de la prestación y su gestión. Existen demasiados organismos implicados con diferentes criterios que hacen difícil el consenso en la gestión de IT. En esta materia confluyen aspectos sanitarios esenciales con otros no menos importantes como son los laborales, económicos y sociales. Los profesionales sanitarios toman decisiones clínicas que conllevan una prestación económica de gran trascendencia cuanti-

\section{BIBLIOGRAFÍA}

1. Simón P. "La ética de las organizaciones sanitarias: el segundo estadio de desarrollo de la bioética" Rev Calidad Asistencial. 2002; 17 (4): 247-59.

2. Junta de Andalucía, Consejería de Salud, Sistema Sanitario Público de Andalucía. II Plan de Calidad. Caminando hacia la excelencia. 2005-2008.

3. Jefatura del Estado. Ley 16/2003 de 28 de mayo, de cohesión y calidad del Sistema Nacional de Salud. BOE n. 128, 20567-20588.

4. Junta de Andalucía, Consejería de Salud, Sistema Sanitario Público de Andalucía. I Plan de Calidad. Nuevas Estrategias para la Sanidad Andaluza. 2000-2004. tativa y deben estar muy implicados en la gestión de unos recursos que son limitados (24).

Destacamos la falta de formación de los profesionales sanitarios responsables de la prescripción de la prestación de IT, desde el pregrado hasta el postgrado no hay asignaturas en las que se trate específicamente de la gestión de la Incapacidad Temporal. Las decisiones clínicas deben estar basadas en la mejor evidencia científica disponible y en la valoración de la alteración de la función y estructura corporal, relacionada con la limitación de la actividad.

En el programa específico de Plan de Mejora de Atención al Usuario se encuentra la formación en Incapacidad Temporal a los facultativos de Atención Primaria de Salud. La plataforma virtual ideada desde nuestra Unidad (e-FormIT), reconocida en Congresos Nacionales, es una herramienta valiosa para una formación práctica inicial y continuada que sirva para mejorar la atención de los ciudadanos asistidos por los Equipos Básicos de Atención Primaria. A la vez que implica a los compañeros del primer nivel de asistencia sanitaria en la gestión de la Incapacidad Temporal.

Existe unanimidad respecto a la necesidad de unificar criterios de gestión de la Incapacidad Temporal, por lo que sería interesante consensuar este documento con el resto de Unidades Médicas de Valoración de Incapacidad que realizan su trabajo en la Comunidad Autónoma Andaluza, pudiéndose extender a otras Autonomías.
5. Junta de Andalucía, Consejería de Salud. Decreto 224/2005, de 18 de octubre, por el que se aprueba el Reglamento de Ordenación de la Inspección de Servicios Sanitarios de la Junta de Andalucía. BOJA n. 217, 24-30.

6. Cortina A, Conill J. "Ética y empresas sanitarias" En: Simón P (Ed) En: La ética de las organizaciones sanitarias. Nuevos modelos de calidad. Madrid: Triacastela; 2004: 1-15.

7. Junta de Andalucía, Consejería de Salud. Orden de 23 de febrero de 2006, por la que se aprueba el Plan Anual de Inspección de Servicios Sanitarios. BOJA n. 52, 15-22. 
8. Agencia de Calidad Sanitaria de Andalucía, Consejería de Salud. Programa de Acreditación de Centros del Sistema Sanitario de Andalucía. Manual de Estándares (ME 2 1_03), 2004.

9. European Foundation for Quality Management. European Excellence Model. Brussels, 2000. Disponible en: http//www.efqm.org.

10. Pérez Lázaro JJ, García Alegría J, Tejedor Fernández M. Gestión clínica: conceptos y metodología de aplicación. Rev Calidad Asistencial 2002;17:305-11.

11. Lorenzo S. "Evidencias de aplicación del Modelo EFQM de Excelencia al sector sanitario en nuestro medio" Rev Calidad Asistencial. 2000; 15: 129-30.

12. Donabedian A "Evaluación de la calidad en la atención médica" Rev Calidad Asistencial 2001; 16: S11-S27

13. Tejedor, M., Pérez, J.J., García, J. Gestión Clínica: aplicación práctica en una unidad hospitalaria (II). Rev. Calidad Asistencial 2003; 18 (2): 125-31.

14. Arbusa, I, Alfaro, L, Zamora, J.J. El Sistema Nacional de Salud en el siglo XXI. La gestión de calidad en la provisión de servicios sanitarios. Rev. Administración Sanitaria. 2005; 3(3):431-46.

15. Ministerio de Administraciones Públicas. Real Decreto 951/2005, de 29 de julio, por el que se establece el marco general para la mejora de la calidad en la Administración General del Estado. BOE n. 211, 30204-30211.

16. Arcelay A, Lorenzo S, Bacigalupe M, Mira JJ, Ignacio E, Palacio F, et al. Adaptación de un modelo de calidad total a las instituciones sanitarias españolas. Rev Calidad Asistencial 2000;25:184-92.
17. Mira, J.J, Gómez, J.M., Blaya, I., García, A. Universidad Miguel Hernández de Elche. La gestión por Procesos.

18. Temes, JL. Gestión de procesos asistenciales. Aplicación práctica. (Editorial McGraw-Hill), 2006.

19. Inspección de Trabajo y Seguridad Social. Estudio sobre indicadores de eficacia de la Inspección de Trabajo y Seguridad Social. ITSS 2006 www.mtas.es/itss/que_hacemos/doc/sgi-estudio-de-indicadores-eficacia.ppt

20. Davins J. "Construcción y análisis de indicadores para monitorizar la calidad" En Tratado de Calidad Asistencial en Atención Primaria. 1997 Ed. Dupont Pharma: 251-268

21. Joint Commission International Accreditation y Fundación Avedis Donabedian Estándares Internacionales de Acreditación de Hospitales. Barcelona: Joint Commission International Accreditation y Fundación Avedis Donabedian, 2001

22. Donabedian A "Los siete pilares de la calidad" Rev Calidad Asistencial 2001; 16: S96-S100.

23. Irigoyen J. "La perspectiva cultural en la gestión del cambio" En Silió-Villamiñ F, Manuel-Keenoy E (Ed). En: La gestión del cambio en los servicios sanitarios. 1997 Ed. Escuela Andaluza de salud Pública. Granada: $109-14$.

24. Lázaro y de Mercado P. "Motivación, incentivos, eficiencia y ética en los sistemas de salud" En Técnicas Avanzadas de Investigación en Servicios de Salud. Madrid 2001. 